\title{
What Is New in LATEX? VI. LTEX on an iPad. Empire
}

\author{
G. Grätzer
}

\section{Introduction}

In the first part of this article, Foundation (Notices, March 2013), I hope I convinced you that the iPad is a computer. Now we proceed to discuss some LATEX implementations on this computer.

\section{With Two Hands behind Your Back}

To implement LTEX on an iPad, two major manmade obstacles have to be overcome: Sandboxing and the GPL license.

\section{Sandboxing}

We discussed this topic in Foundation. Does it impact LTEX implementations? You bet.

For instance: The LATEX implementation Texpad on the Mac is given a single $\mathrm{LTEX}$ root file. It then reads through the LATEX source, gets all the included files, and presents you with an outline of your project. Sandboxing would not allow this. The handling of the auxiliary files also poses a problem. Of course, these problems can be overcome by ingenious programmers.

\section{GPL}

Richard Stallman, ${ }^{1}$ of Emacs fame, started the GNU operating system in 1983. Soon after, he started a nonprofit corporation called the Free Software Foundation.

Stallman wrote, with the assistance of some law professors, the General Public License (GPL) - the most widely used free software license-released in 1989. Version 3 is dated June 29, 2007, the day the

G. Grätzer is Distinguished Professor of Mathematics, retired, at the University of Manitoba. His email address is gratzer@me.com.

${ }^{1}$ Picture taken from his personal website. DOI: http://dx.doi.org/10.1090/noti963

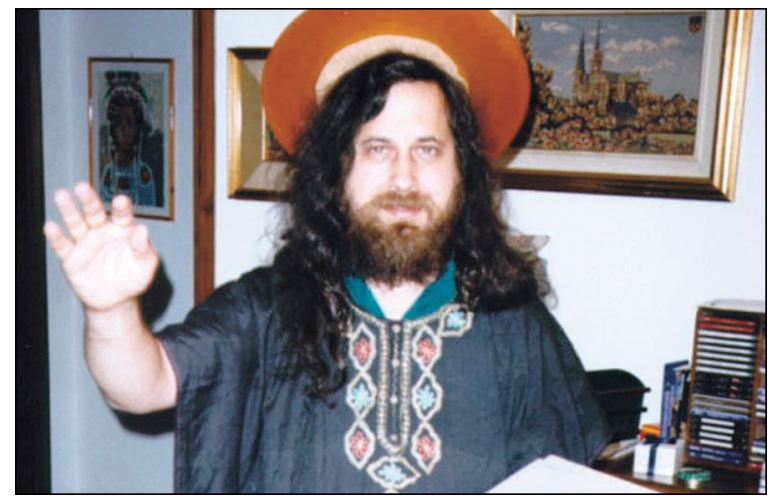

Richard Stallman

iPhone was released. Many software developers use GPL to ensure the free distribution of their software (source code and executable) under reasonable terms.

Some software developers seem not to be aware of the fact that GPL-licensed software cannot be used in an app created for the iPad. Two wellknown developers explained to me that they use GPL because their peers do. Both would like to get out of it but do not know how.

How ironic: the license that was supposed to allow you to spread your free software to wherever it is needed now stops you from having it used on the fastest-growing platform of all time.

\section{The 28 Flavors...}

The iPad LTEX implementations differ, naturally, in almost all aspects, but we can get a crude classification by specifying how they obtain the LTEX files and how they typeset them. 


\section{Where Are You Going?}

The LATEX source files, of course, can always be composed in the app, but typically you already have them. You can obtain your existing source files in two ways:

1. Using iTunes. To transfer files-one at a timeto your app from your computer using iTunes, connect your iPad to your computer and start iTunes by double-clicking on its icon.

Under Devices, select the iPad from the left side of the iTunes window; see Figure 1.

At the top of the iTunes window, next to Summary and Info, select Apps; see Figure 2.

The lower part of the window now has File Sharing; see Figure 3.

On the left you see a listing of the apps available for file transfer. Select the app; the files already in the app are then listed in the right pane. Click on the add button, and a file browser appears. Choose the file you want to transfer.

2. Via Dropbox. I assume that you have the ubiquitous Dropbox (the application that keeps your files safe and up to date across multiple devices and platforms). For an introduction, go to dropbox.com/gs.

In the app you sign in to Dropbox. Now the app can see the contents of your Dropbox or some part of it (at the Dropbox server) as long as you have an Internet connection.

\section{And What Do You Wish?}

The app may typeset the LTEX file in the following ways:

A. On Your iPad. This is the "post-PC revolution" option: the app places a LTEX distribution on the iPad and you typeset with it. No computer or Internet connection is required.

However, a complete IATEX distribution is about 4 GB! No app can be this big. So you only get a minimal LATEX distribution and no special fonts!

B. On Your Computer via Dropbox. This is the most powerful option. You have all the packages and fonts on your computer available to you. An app (such as AutomaTeX by Jonathan Weisberg) monitors if there is any change in the LATEX file in Dropbox. If there is, the file is retypeset and the PDF is made available to you via Dropbox.

C. In the Cloud. This option provides you with a remote server, the Cloud; you connect to it with Wi-Fi. The server has a full LATEX implementation, so you miss only the special fonts. And, of course, you must have Wi-Fi to use it. So you cannot polish up your lecture on the airplane on the way to a meeting.

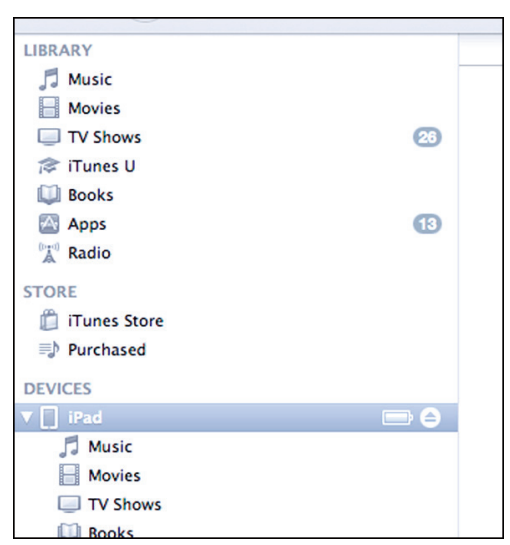

Figure 1. Under Devices, we selected the iPad.

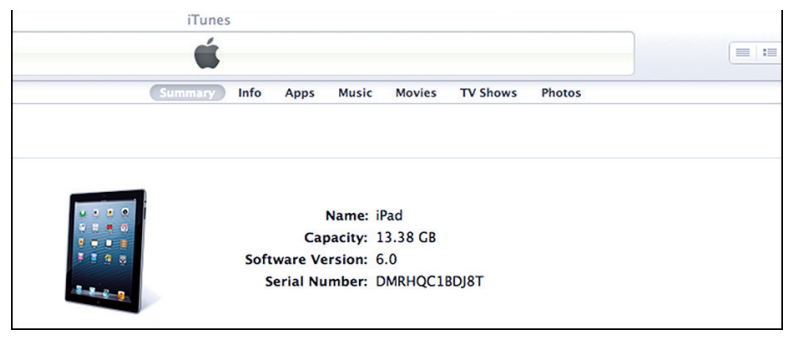

Figure 2. Choose Apps.

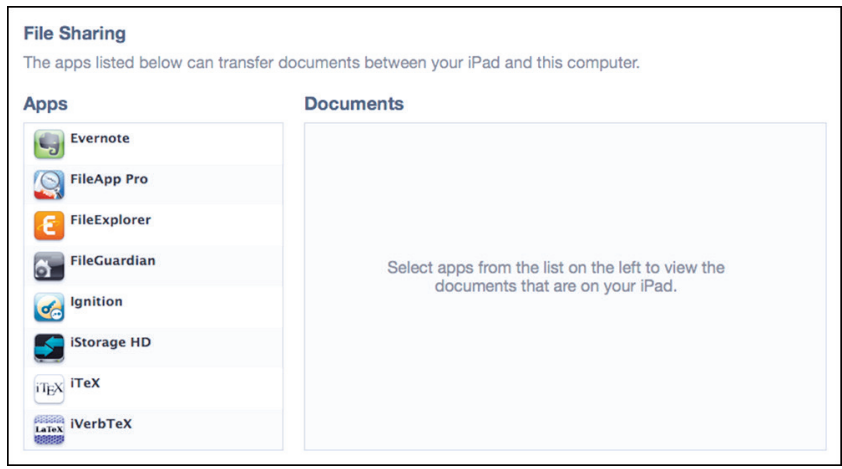

Figure 3. Select app.

\section{What Do You Get?}

Originally, LATEX output was a dvi file. These days, utilizing pdftex (under GPL license) by Hàn Thế Thành, the output is PDF. Since developers could not use GPL-d code, the output was dvi. These days, even on the iPad, PDF rules. In a more perfect world, these talented developers would not have to spend so much time reinventing GPL-d wheels.

\section{Keyboard or Not to Keyboard...}

In Figure 4 you see editing with the iPad's soft keyboard (notice the extra row of IATEX keys added by the LTEX implementation Texpad) and in Figure 5, editing with a Bluetooth keyboard (notice that the extra row of LATEX keys of Texpad is still present). 


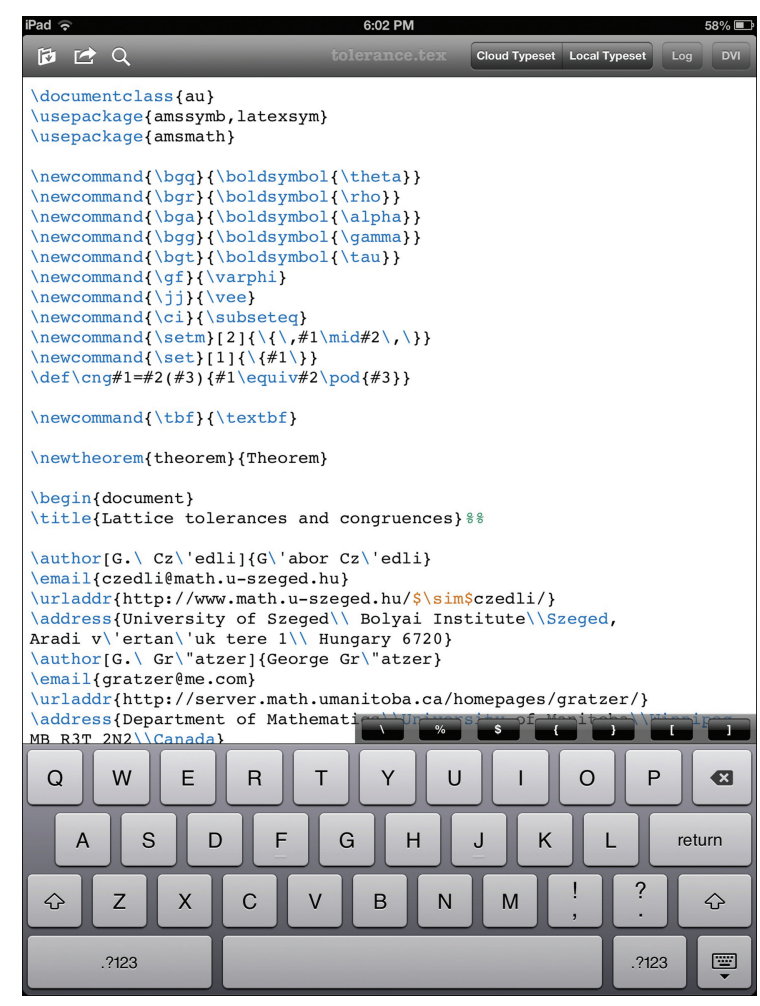

Figure 4. Editing with soft keyboard.

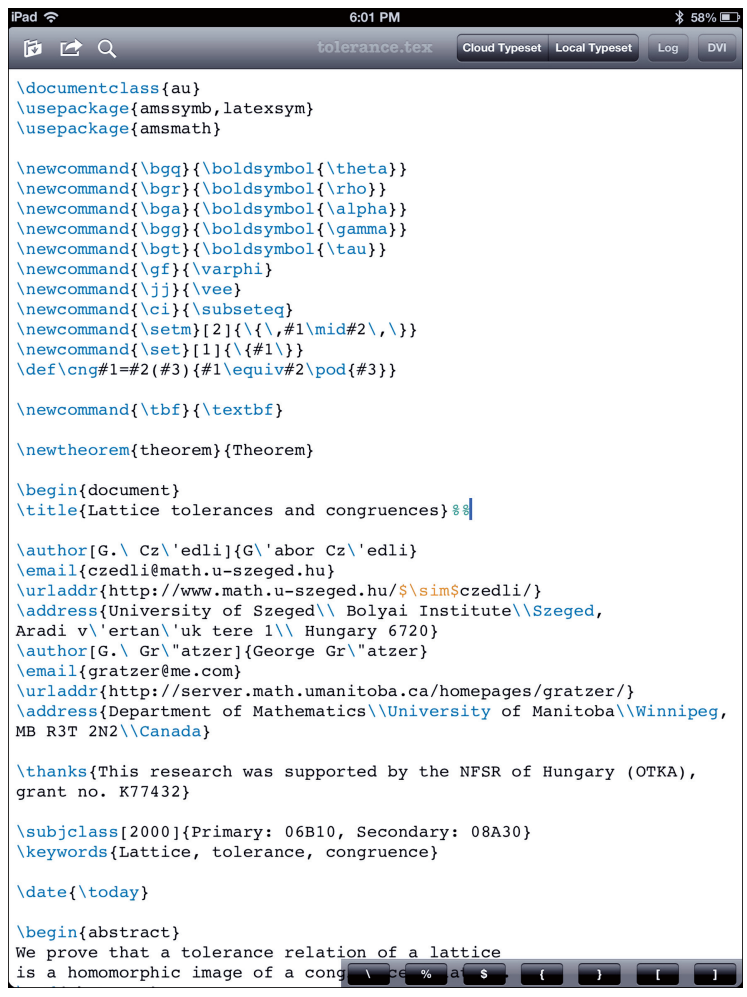

Figure 5. Editing with Bluetooth keyboard.

\section{Two "Post-PC Revolution" LATEX Implementations}

We now discuss the two LTEX implementations that typeset on the iPad.

\section{Texpad}

Files: 2. Typesetting: A, B, C.

Documentation: Excellent and detailed on the iPad interface. It is available as a help file and also at http://texpadapp.com/app-help-files/ios/help.htm7.

Samuel Johnson famously recommended that the introduction of a book should set out the goal, explain why it cannot be done, and then proceed to accomplish it. Valletta Ventures's Duncan P. Steele and Jawad A. Deo took this to heart. They wrote blogs explaining why there can be no LATEX on the iPad and then proceeded to implement it.

Texpad is a LATEX implementation for the Mac and for the iPad. It has some interesting features, including:

- Autocompletion of all common commands and autofilling \cite-s and \ref-s.

- Replacement of the IATEX console with a list of errors and warnings linked to the source.

- Global search, outline view, and syntax highlight.

Step 1. To get started with Texpad, go to the iPad App Store and install Texpad.

Step 2. Now open Texpad. Figure 6 shows Texpad at the first startup.

The Help button gets the help file.

Step 3. Touch Off to turn Dropbox on. Sign up for Dropbox with the same email address and password as for your computer's Dropbox. (If you have Dropbox installed and connected, it's even simpler; you just have to Allow the connection.) Your File Storage now gives two options: iPad and Dropbox; see Figure 7.

It is important to understand that your LATEX files will live in the Dropbox (in the Cloud, at the Dropbox server) or locally on your iPad.

Step 4. The Dropbox files are now available to you by touching Dropbox under File Storage; see Figure 7.

- First, create a folder for the LTEX files to be transferred. Navigate to iPad file storage. Touch the + in the bottom right and choose Folder. Name the folder.

- Second, navigate to the Dropbox file system view and to the folder containing the file you want to copy. Touch Edit. Select the file to transfer. At the bottom center, touch Copy. Navigate to the folder into which you want to copy the file and touch Copy.

Step 5. Typesetting will take place either on the iPad or in the Cloud.

Go to the folder of a LATEX file, touch the file (on the iPad or in the Dropbox), and typeset it on the iPad (touch Local Typeset) or in the Cloud, that is, at Valletta's server (touch Cloud Typeset).

Step 6 . Try to visualize what is happening.

$6 \mathrm{a}$. If you typeset on the iPad and the file is on the iPad, it just typesets locally; that is it. 


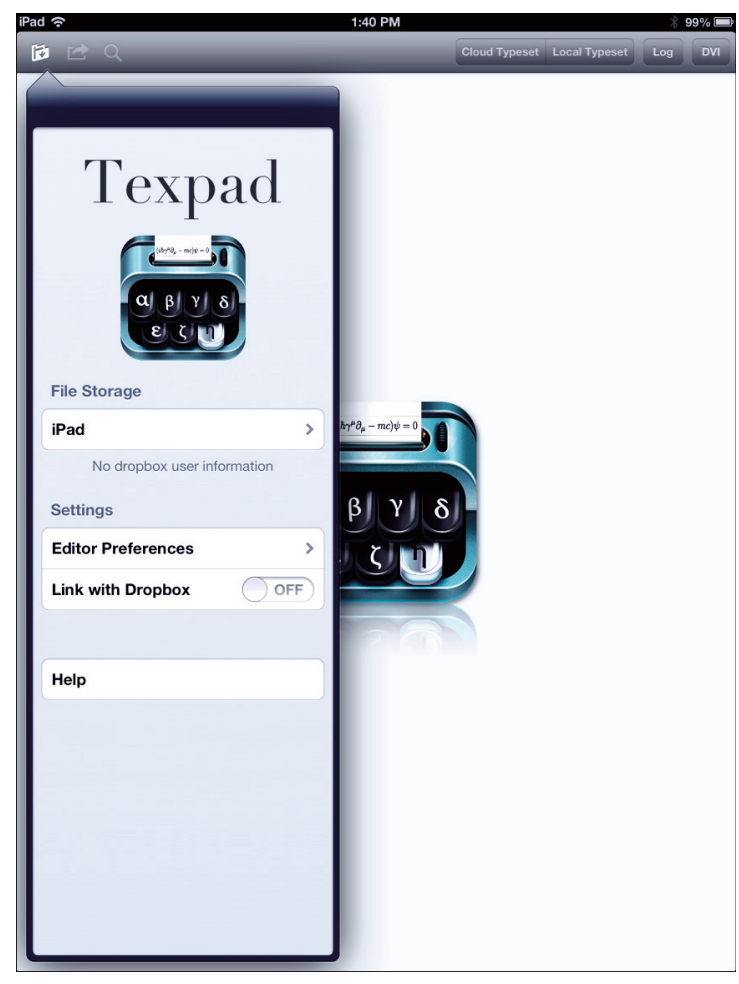

Figure 6. Texpad first startup.

File Storage

\section{iPad}

\section{Dropbox}

Dropbox Account: George Gratzer

Figure 7. Expanded File Storage.

6b. If you typeset on the iPad and the file is in Dropbox, then the file is transferred to the iPad, typeset, and the resulting PDF is sent back to the Dropbox; nothing is kept on the iPad.

6c. If you typeset in the Cloud and the file is in Dropbox, the file is transferred to the Cloud, typeset, and the resulting PDF is sent back to Dropbox; nothing is kept in the Cloud.

$6 \mathrm{~d}$. If you typeset in the Cloud and the file is on the iPad, the file is transferred to the Cloud, typeset, and the resulting PDF is sent back to the iPad; nothing is kept in the Cloud.

Step 7. Once you touch a LTEX file, you are ready to edit it. Cursor control is very important. You do it with a two-finger swipe. Of course, this is not so important if you use a Bluetooth keyboard; it has cursor keys. But two-finger swipe is faster!

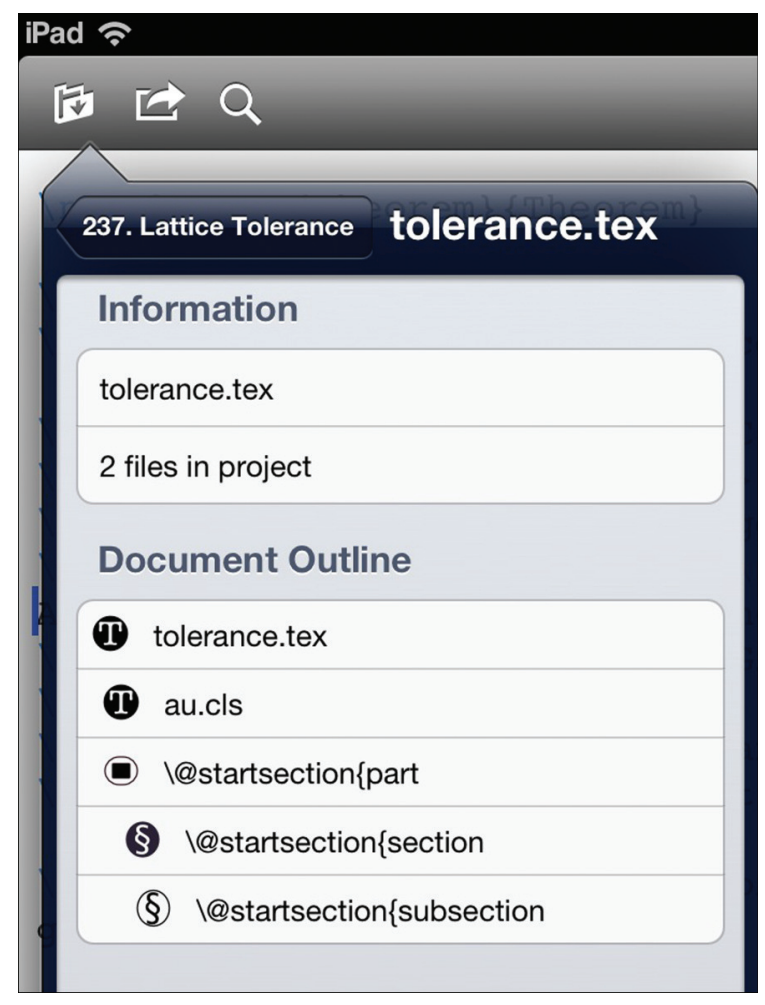

Figure 8. Organizer window.

Step 8. You've edited and typeset your LATEX file. You want to get to another file.

Touch the organize button (the folder icon in the upper left). You get the organizer window; see Figure 8.

Touch the button in the upper left of the window. You get back to Dropbox, eventually to the expanded File Storage of Figure 7.

$$
* * * * * * * * * * *
$$

These eight steps should be enough to get you started. Read the Help file for more information.

\section{TeX Writer}

Files: 2. Typesetting: A.

Documentation: The file readme.pdf is no quick start, but it is useful for understanding how TeX Writer works and how to customize it.

TeX Writer was the first to typeset on the iPad. It could typeset only TeX files. Now it has LTEX and the AMS packages on board.

Step 1 . When you start up TeX Writer, first link to Dropbox.

In TeX Writer you get the display of Figure 9, showing the source file readme.tex. Pressing the More icon (right-pointing arrow), you get more icons to read the PDF version or Air Printing readme.pdf. 


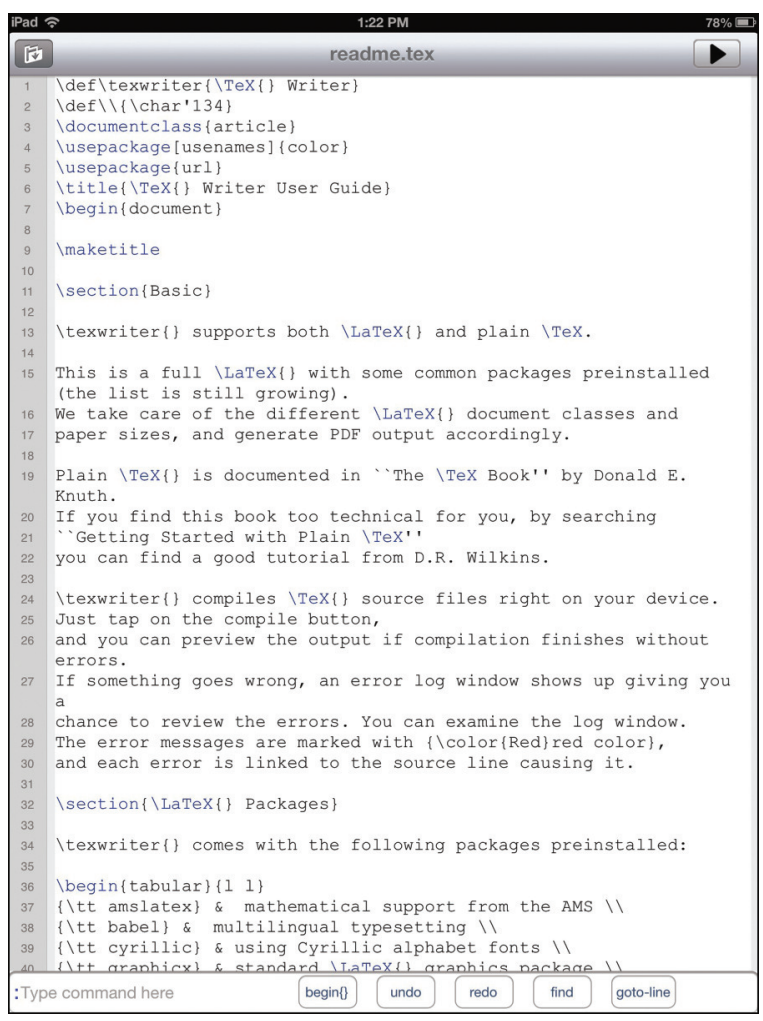

Figure 9. TeX Writer startup.

\begin{tabular}{|l|l|}
\hline Name \\
\hline Apps \\
\hline TeX Writer \\
D readme.tex \\
Camera Uploads \\
Driver evaluation.pdf \\
Family Folder \\
2 iPad-Superguide-Third-Edition \\
LaTeX on IPad \\
Lattice Theory STA \\
\hline
\end{tabular}

Figure 10. Dropbox folders.

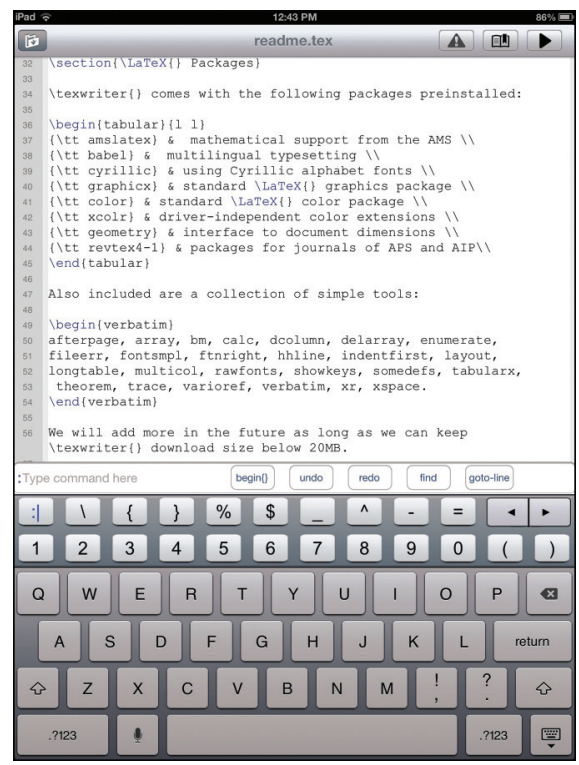

Figure 11. Typing in TeX Writer.
On the left is the organize icon; touching it, you get a file listing: readme.tex and readme.pdf. At the bottom is New File; touch it to compose one.

Step 2. So you are perplexed about what to do next; you've run out of icons. You have to know that TeX Writer accesses Dropbox in a special way.

When you connect to Dropbox from TeX Writer, it creates a new folder App in Dropbox. In App it creates the subfolder TeX Writer; see Figure 10 . In this subfolder you find readme.tex. Anything you put in the TeX Writer subfolder is visible in the file listing window on the iPad; anything not in this subfolder is not visible to TeX Writer.

Step 3. TeX Writer gets your files from this subfolder in Dropbox. Place in there a folder with the files of your current project. These will be available to you on your iPad. Moreover, these files are fully synchronized, so the editing changes you make on your iPad show up in Dropbox.

Step 4. LATEXing, you spend most of your time editing. TeX Writer's editor has some interesting features, for instance, excellent cursor control; touch begin $\{$, type in the name of the environment, and the environment is placed in your article; undo, redo, search, and so on; see Figure 11.

Note that, when typing, you retain the editing functions you see in Figure 9 and, in addition, you get an extra row of LATEX-specific keys. You do not get them with a Bluetooth keyboard; however, the keyboard may have many of these keys.

Note that the Log viewer links to error lines.

\section{Other TEX -Related Apps}

The editor Textastic (see Foundations) has Dropbox connection and it is LATEX aware, so it can be viewed as a LATEX implementation.

Now we present two LATEX implementations that are not of the "post-PC revolution".

\section{Tex Touch}

Files: 2. Typesetting: A, B.

Documentation: Three videos and a quick start guide.http://www. vancapy.com/textouch/

Step 1. Sign up at TeX Cloud as instructed.

Step 2. Touch Dropbox and sign in for your Dropbox account.

Step 3. A window comes up to set the tex folder in Dropbox. Navigate to the folder that has your LTEX project; touch Set TeX folder.

Step 4. Touch download for all the files you need. The downloaded files appear in the left pane, each with an upload button. Touch them to upload the files to the TeX Cloud.

They appear in the left pane. Touch Done.

Step 5. The display of Figure 12 comes back, except that now it has a right pane listing the files in the Cloud. Touch your texfile and an important 


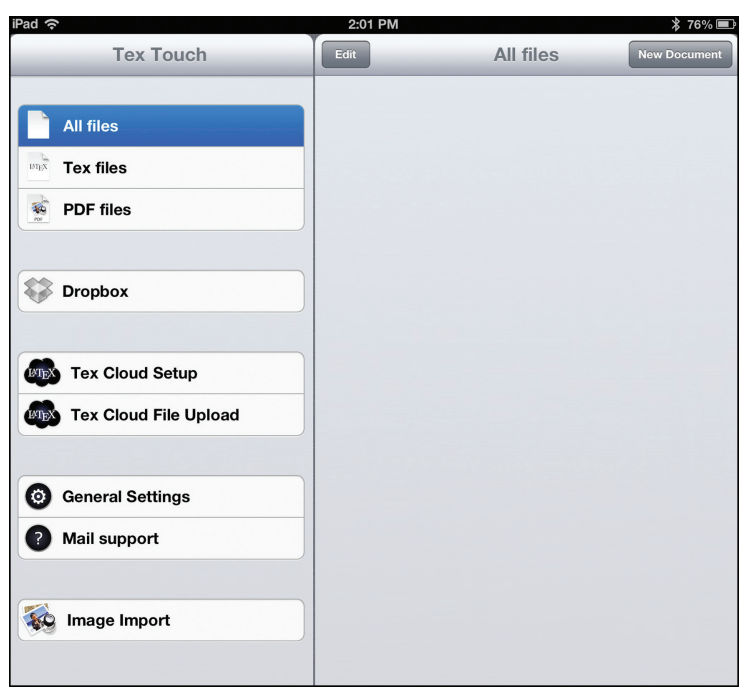

Figure 12. Starting up with TeX Touch.

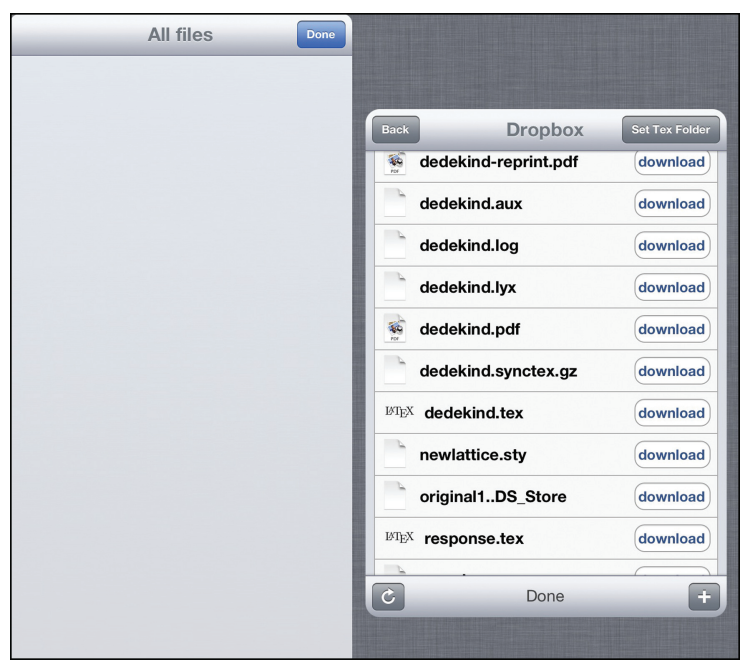

Figure 13. The Dropbox interface.

hint comes up: when editing, one finger swipe moves the cursor one character! Then the file comes up for editing. Touch the text anywhere, and the usual iPad keyboard comes up, augmented with a scrolling keyboard row of keys important for LTEX; these symbols are also displayed if you use a Bluetooth keyboard.

Step 6. Once you are done editing, touch TeX in the upper right corner, then the TeX! in the upper left corner. The file gets uploaded to the Cloud, typeset in the Cloud, and the PDF file gets downloaded to the iPad. Touching PDF displays the PDF file. Exit by touching Done, then Document.

\section{iVerbTeX}

Files: 1. Typesetting: C.

Documentation: None that I could find.

iVerbTeX stores your documents locally on your iPad or in the Cloud. You edit locally. The text editor is the standard iPad text editor, so it is not suitable for editing tex files.

There are some more apps that use $\mathrm{TEX}$ and LTEX. Here are two:

\section{iTeX}

In Project Gutenberg ${ }^{1}$ or arXiv.org, ${ }^{2}$ select a document, and iTeX will attempt to write the LTEX code of a nicely formatted LATEX output for the iPad's size and resolution.

\section{TeX Equation}

This app typesets math formulas with its built-in TEX engine for use in Pages and Keynote.

\section{Conclusion}

So this is my new best friend for the road, my iPad with the Ultrathin Keyboard Cover for iPad by Logitech.

How many times have you read reviews of words processors concluding with "And I wrote this review with..." Well, I did not. This article uses the Notices style, requiring the Lucida fonts, and font installations are very difficult on the iPad.

So LATEXing on an iPad requires some compromises. Nevertheless, when not at your desk, the iPad will be nearly as functional as your MacBook Air, and it is so much easier to carry around.

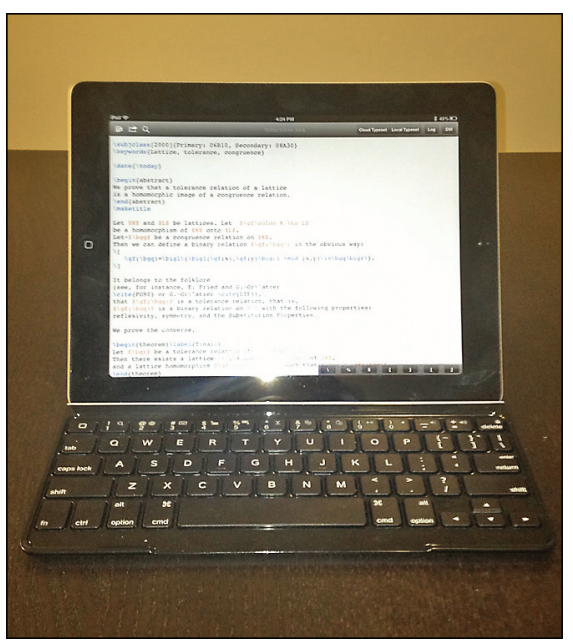

My new best friend.

\footnotetext{
${ }^{1}$ An archive of over 40,000 public domain eBooks.

${ }^{2}$ An archive of over 800,000 scientific e-prints.
} 